\title{
Optical binding of magnetodielectric Rayleigh particles
}

\author{
Patrick C. Chaumet ${ }^{1}$ and Adel Rahmani ${ }^{2,3}$ \\ ${ }^{1}$ Institut Fresnel (UMR 7249), Ecole Centrale de Marseille, Aix-Marseille Université, Av. Escadrille Normandie-Niemen, \\ 13397 Marseille Cedex 20, France \\ ${ }^{2}$ School of Mathematical Sciences, University of Technology Sydney, Broadway NSW 2007, Australia \\ ${ }^{3}$ Ecole Centrale Marseille, 38 rue Frédéric Joliot-Curie, 13451 Marseille Cedex 20, France
}

(Received 5 February 2013; published 20 May 2013)

\begin{abstract}
We present a theoretical and numerical study of the optical binding and optical torque between two Rayleigh particles with arbitrary, complex, scalar dielectric permittivity and magnetic permeability. We use a computational approach based on the discrete dipole approximation to derive the optical force and torque experienced by the particles when illuminated by a linearly or circularly polarized plane wave. We show that optical binding between magnetodielectic particles is qualitatively different from the traditional case involving dielectric particles only. In particular, we show that for certain configurations, the system of two magnetodielectric particles will experience a long-range optical torque whose amplitude envelope does not decay with the separation between the particles.

DOI: 10.1103/PhysRevB.87.195125

PACS number(s): 42.50.Wk, 42.25.Fx, 41.20.-q
\end{abstract}

\section{INTRODUCTION}

Since Ashkin demonstrated that optical fields can produce forces on neutral particles, ${ }^{1}$ electromagnetic forces and torques have been used in a broad variety of applications, from atom cooling ${ }^{2-4}$ to the optical trapping and manipulation of macroscopic samples, such as biological cells. ${ }^{5-8}$ For particles smaller than the trapping wavelength, there exist several nano-optical tweezers techniques, usually exploiting the strong gradient force associated with the evanescent field present in the near field of a subwavelength scatterer. ${ }^{9-13}$ Advanced holographic techniques have allowed traditional optical tweezers approaches to be extended to the trapping and manipulation of multiple particles, of sizes comparable to the wavelength. ${ }^{7}$ For smaller particles, however, multiple trapping is much more challenging because of the difficulty in creating multiple traps that are packed closely enough and yet distinct. Traditional beam-shaping techniques, using lenses and spatial filters, must generally be abandoned for more "integrated" approaches where a photonics structure, such as an array of scatterers or a photonics crystal, is used to generate a highly inhomogeneous optical field, with strong enough optical gradients to induce trapping. ${ }^{14-18}$

There is, however, another strategy for the multiple trapping of small particles (i.e., small compared to the wavelength of light used): instead of trying to generate multiple trapping sites that exist independently of the trapped particles, one can exploit optical binding. The possibility of optically induced bound states was first pointed out by Burns et al. ${ }^{19}$ This opened the way to the development of optically induced microstructure assembly techniques in two- or three-dimensional arrangements. ${ }^{20-22}$ The canonical problem of the optical binding between two particles has been studied extensively for dielectric spheres in homogeneous space ${ }^{23-25}$ or in the presence of a dielectric substrate. ${ }^{26}$ See Ref. 27 for a review. Optically induced torque has been also studied between bounded spheres. ${ }^{28,29}$

Over the past decade, metamaterial research has highlighted how the relative permittivity and permeability of structure can be controlled by a careful design of the material composition and geometry of the structure. Thus, one can create a metamaterial with, for example, a double-negative ${ }^{30}$ or a double-positive electromagnetic response. ${ }^{31}$ This type of metamaterial, constructed from a clever design of electromagnetic elements whose tailored electromagnetic responses, when taken collectively, yield an effective permittivity and an effective permeability which can not be found in nature. ${ }^{32}$ However, some metamaterial responses can be engineered in a simpler way, as emphasized in Ref. 33, where small dielectric particles, made of nonmagnetic materials can present scattering properties similar to those of magnetodielectric particles. Hence, it is interesting to study how a magnetic response can affect quantitatively, but most importantly qualitatively, the properties of optical forces and torques. ${ }^{33-36}$ Already, the possibility of generating negative optical forces using metamaterials has been discussed in Ref. 37.

In this paper, we present a general description of the canonical problem of the optical binding and optical torque between two magnetodielectric (MD) Rayleigh particles. The analytic expression of the force and torque on the particles is derived for several configurations, and is validated numerically with the discrete dipole approximation. In Sec. II, we derive the general expressions of the optical binding force and the optical torque between two MD Rayleigh spheres. In Sec. III, we discuss the general features of optical binding of MD spheres under linearly and circularly polarized illuminations. In particular, we show that, under certain conditions, it is possible for the system of two spheres to experience a longrange optical torque that does not decay with the separation of the spheres. In Sec. IV, we present our conclusion.

\section{GENERAL EXPRESSIONS FOR THE ELECTROMAGNETIC FORCE AND TORQUE FOR TWO COUPLED RAYLEIGH PARTICLES}

Consider two Rayleigh particles (spheres) located at positions $\mathbf{r}_{1}$ and $\mathbf{r}_{2}$ and illuminated by an incident electromagnetic field $\left\{\mathbf{E}^{\text {inc }}, \mathbf{H}^{\text {inc }}\right\}$. Let $\mathbf{T}$ denote the electromagnetic field susceptibility tensor, and $\alpha^{\mathrm{e}}$ and $\alpha^{\mathrm{m}}$ the electric and magnetic polarizabilities of a dipolar sphere. For simplicity, we assume 
that the background medium is vacuum. The self-consistent local field at the position of sphere 1 can be written as

$$
\begin{aligned}
\mathbf{E}\left(\mathbf{r}_{1}\right)= & \mathbf{E}^{\mathrm{inc}}\left(\mathbf{r}_{1}\right)+\mathbf{T}^{\mathrm{ee}}\left(\mathbf{r}_{1}, \mathbf{r}_{2}\right) \alpha^{\mathrm{e}}\left(\mathbf{r}_{2}\right) \mathbf{E}\left(\mathbf{r}_{2}\right) \\
& +\mathbf{T}^{\mathrm{eh}}\left(\mathbf{r}_{1}, \mathbf{r}_{2}\right) \alpha^{\mathrm{m}}\left(\mathbf{r}_{2}\right) \mathbf{H}\left(\mathbf{r}_{2}\right), \\
\mathbf{H}\left(\mathbf{r}_{1}\right)= & \mathbf{H}^{\mathrm{inc}}\left(\mathbf{r}_{1}\right)+\mathbf{T}^{\mathrm{he}}\left(\mathbf{r}_{1}, \mathbf{r}_{2}\right) \alpha^{\mathrm{e}}\left(\mathbf{r}_{2}\right) \mathbf{E}\left(\mathbf{r}_{2}\right) \\
& +\mathbf{T}^{\mathrm{hh}}\left(\mathbf{r}_{1}, \mathbf{r}_{2}\right) \alpha^{\mathrm{m}}\left(\mathbf{r}_{2}\right) \mathbf{H}\left(\mathbf{r}_{2}\right),
\end{aligned}
$$

with similar expressions for sphere 2 obtained by the exchange of indices 1 and 2. The superscript (e or h) of $\mathbf{T}$ indicates the the electric or magnetic nature of the source and the field. From these expressions, the electric and magnetic fields at each sphere can be found by solving analytically a linear system of size $12 \times 12$. To compute the electromagnetic force on sphere 1 , we also need the derivative of the electromagnetic field at $\mathbf{r}_{1}$ which an be derived from Ref. 38 as

$$
\begin{aligned}
\partial_{i} \mathbf{E}\left(\mathbf{r}_{1}\right)= & \partial_{i} \mathbf{E}^{\mathrm{inc}}\left(\mathbf{r}_{1}\right)+\partial_{i} \mathbf{T}^{\mathrm{ee}}\left(\mathbf{r}_{1}, \mathbf{r}_{2}\right) \alpha^{\mathrm{e}}\left(\mathbf{r}_{2}\right) \mathbf{E}\left(\mathbf{r}_{2}\right) \\
& +\partial_{i} \mathbf{T}^{\mathrm{eh}}\left(\mathbf{r}_{1}, \mathbf{r}_{2}\right) \alpha^{\mathrm{m}}\left(\mathbf{r}_{2}\right) \mathbf{H}\left(\mathbf{r}_{2}\right), \\
\partial_{i} \mathbf{H}\left(\mathbf{r}_{1}\right)= & \partial_{i} \mathbf{H}^{\mathrm{inc}}\left(\mathbf{r}_{1}\right)+\partial_{i} \mathbf{T}^{\mathrm{he}}\left(\mathbf{r}_{1}, \mathbf{r}_{2}\right) \alpha^{\mathrm{e}}\left(\mathbf{r}_{2}\right) \mathbf{E}\left(\mathbf{r}_{2}\right) \\
& +\partial_{i} \mathbf{T}^{\mathrm{hh}}\left(\mathbf{r}_{1}, \mathbf{r}_{2}\right) \alpha^{\mathrm{m}}\left(\mathbf{r}_{2}\right) \mathbf{H}\left(\mathbf{r}_{2}\right),
\end{aligned}
$$

where $\partial_{i}$ is the derivative with respect to $\mathbf{r}_{1}$ of field component $i=x, y$, or $z$. Note that here, in contrast to the fields, the spatial derivatives of the fields are not calculated in a self-consistent way.

With the total electromagnetic fields and their spatial derivatives known, component $u$ of the electromagnetic force experienced by sphere 1 can be written as

$$
\begin{aligned}
& F_{u}\left(\mathbf{r}_{1}\right) \\
& =\frac{1}{2} \operatorname{Re}\left[\alpha^{\mathrm{e}}\left(\mathbf{r}_{1}\right) E_{v}\left(\mathbf{r}_{1}\right) \partial_{u} E_{v}^{*}\left(\mathbf{r}_{1}\right)+\alpha^{\mathrm{m}}\left(\mathbf{r}_{1}\right) H_{v}\left(\mathbf{r}_{1}\right) \partial_{u} H_{v}^{*}\left(\mathbf{r}_{1}\right)\right. \\
& \left.\quad-\frac{2 k_{0}^{4}}{3} \epsilon_{u v w} \alpha^{\mathrm{e}}\left(\mathbf{r}_{1}\right) E_{v}\left(\mathbf{r}_{1}\right)\left[\alpha^{\mathrm{m}}\left(\mathbf{r}_{1}\right) H_{w}\left(\mathbf{r}_{1}\right)\right]^{*}\right]
\end{aligned}
$$

where $u, v$, or $w$ stands for either $x, y$, or $z, *$ denotes complex conjugation, and $\epsilon_{u v w}$ is the Levi-Civita tensor. Similarly, the optical torque experienced by sphere 1 can be expressed as

$$
\begin{aligned}
\boldsymbol{\Gamma}\left(\mathbf{r}_{1}\right)= & \mathbf{r}_{1} \times \mathbf{F}\left(\mathbf{r}_{1}\right)+\frac{1}{2} \operatorname{Re}\left\{\left(\left[\alpha^{\mathrm{e}}\left(\mathbf{r}_{1}\right) \mathbf{E}\left(\mathbf{r}_{1}\right)\right]^{*}\right.\right. \\
& \times\left[1+\frac{2}{3} i k_{0}^{3} \alpha_{0}^{\mathrm{e}}\left(\mathbf{r}_{1}\right)\right]^{-1} \mathbf{E}\left(\mathbf{r}_{1}\right)+\left[\alpha^{\mathrm{m}}\left(\mathbf{r}_{1}\right) \mathbf{H}\left(\mathbf{r}_{1}\right)\right]^{*} \\
& \left.\left.\times\left[1+\frac{2}{3} i k_{0}^{3} \alpha_{0}^{\mathrm{m}}\left(\mathbf{r}_{1}\right)\right]^{-1} \mathbf{H}\left(\mathbf{r}_{1}\right)\right)\right\},
\end{aligned}
$$

where $\alpha_{0}^{\mathrm{e}}$ and $\alpha_{0}^{\mathrm{m}}$ are the electric and magnetic polarizabilities without the radiation reaction term, i.e., the polarizabilities deduced from the Clausius-Mossotti relation. ${ }^{39}$ Notice that in Eq. (6) terms of the form $1+\frac{2}{3} k_{0}^{3} \alpha$ are added to satisfy conservation of angular momentum. ${ }^{40-43}$ Naturally, similar expressions for the optical force and torque can be written for sphere 2. The optical torque can be separated into two contributions: the extrinsic part [the first term, $\mathbf{r} \times \mathbf{F}$, in Eq. (6)] and the intrinsic part [the remaining terms in Eq. (6)].

The expressions derived above for the force and torque are general and apply to any system of two coupled magnetodielectric spheres. In the remainder of the paper, we discuss certain configurations that help illustrate how the magnetic response of the particles changes the behavior of the electromagnetic

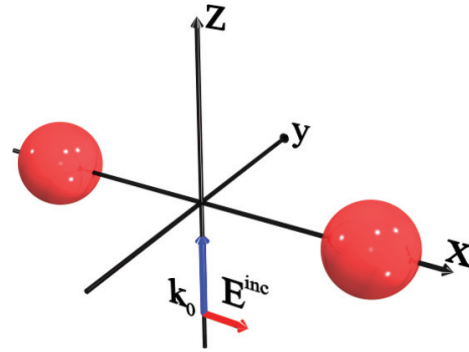

FIG. 1. (Color online) Sketch of the geometry: Two magnetodielectric spheres of radius $r$ illuminated with a plane wave traveling in the positive $z$ direction with wave vector $\mathbf{k}_{0}$.

force and torque, compared to the case of two nonmagnetic particles.

\section{RESULTS AND DISCUSSION}

Figure 1 illustrates the generic geometry of our two-particle system. We choose our system of axes such that the two magnetodielectric spheres, with radius $r$ and relative permittivities (permeabilities) $\left\{\varepsilon_{1}, \varepsilon_{2}\right\}\left(\left\{\mu_{1}, \mu_{2}\right\}\right)$, are initially on the $x$ axis with $\mathbf{a}=\mathbf{r}_{2}-\mathbf{r}_{1}=\mathbf{x}_{2}-\mathbf{x}_{1}, a=|\mathbf{a}|$ and $\mathbf{u}=\mathbf{a} / a$. The system is illuminated with a plane wave $\left\{\mathbf{E}^{\text {inc }}, \mathbf{H}^{\text {inc }}\right\}$ propagating along the $z$ axis and with either linear or left-circular polarization. Note that in this section the optical force and torque are normalized to intensity of the incident field, i.e., $\left|\mathbf{E}^{\text {inc }}\right|^{2}$. The numerical computations are done using the discrete dipole approximation $^{44,45}$ (DDA) where each sphere is represented by 162 subunits, which is a sufficient level of discretization given the small size of the Rayleigh sphere. This discretization is important as it allows us to rigorously take into account the multiple scattering between the two spheres via multipolar orders. This also allows us to analyze the suitability of the dipolar approximation in treating the interaction of the two magnetodielectric Rayleigh spheres. For more details on the computation of electromagnetic forces using the DDA, see Refs. 46-48 for dielectric objects and Refs. 33, 35, and 49-51 for magnetodielectric objects.

\section{A. Symmetric case: Identical particles with $\varepsilon=\mu$}

\section{Illumination with linear polarization}

We first consider two identical Rayleigh spheres with radius $r=\lambda / 60$ and $\varepsilon=\mu$. The spheres are illuminated at wavelength $\lambda$ by a plane wave linearly polarized, and traveling in the positive $z$ direction as depicted in Fig. 1. The general results will be illustrated in the case $\varepsilon=\mu=2$. In Fig. 2, we plot the force experienced by particle 1 versus the separation $a$ between the spheres. Of course, the force experienced by the second particle is exactly the opposite of the force experienced by particle 1 as both spheres are identical. The incident field is linearly polarized along either the $x$ axis (solid line) or the $y$ axis (crosses). Figure 2(a) presents the force in the near-field range, i.e., $a \ll \lambda$ and Fig. 2(b) the force in the far-field range. As shown by Fig. 2, both polarizations give exactly the same optical binding. This effect has been explained intuitively in Ref. 52, but here we focus on the differences compared to the case of two dielectric (i.e., nonmagnetic) particles. 

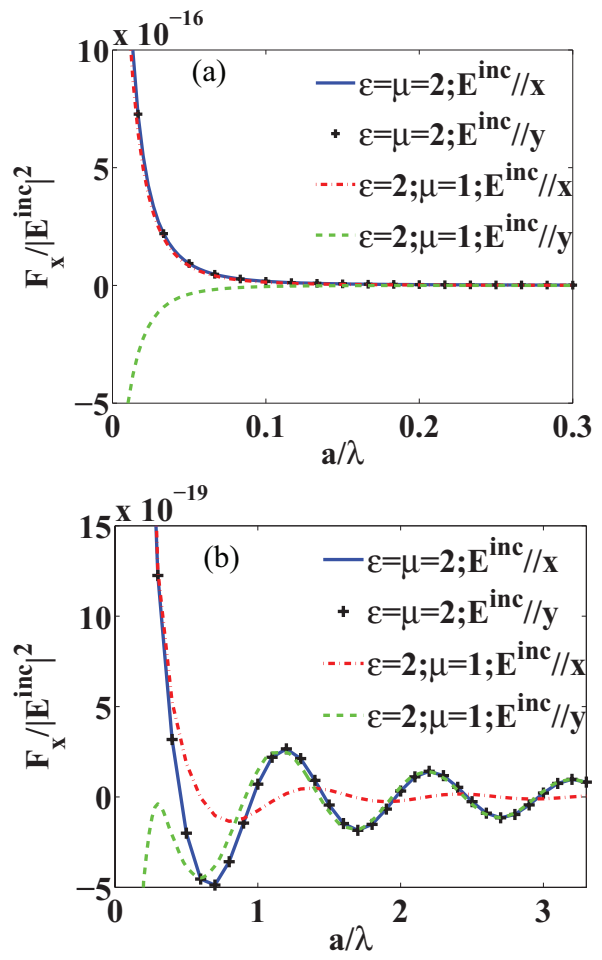

FIG. 2. (Color online) Force experienced by particle 1 for 2 identical spheres with radius $r=\lambda / 60$ and $\varepsilon=\mu=2$. The incident field is linearly polarized along the $x$ axis (solid line) or the $y$ axis (crosses). We also plot the force for 2 dielectric spheres with $\varepsilon=2$ and $\mu=1$, for both polarizations: $x$ axis (dotted dashed line) or $y$ axis (dashed line). (a) Near-field domain; (b) far-field domain.

To gain a better insight into the physics of the problem, we can use Eqs. (1)-(4), along with Eq. (5), to calculate analytically the force on each Rayleigh sphere. However, even in the simple case of two Rayleigh particles, the exact analytical solution of Eq. (5) is not easy to express and interpret. Hence, we further make the assumption that the term $\mathbf{T} \alpha$ is smaller than 1 , which is a valid approximation for Rayleigh particles. ${ }^{26}$ Thus, within this approximation, the field at the location of one of the spheres is the incident field plus the field scattered by the other sphere. Owing to the symmetry of the problem, and the approximation $\mathbf{T} \alpha \ll 1$, there is no component of the field along the $z$ axis. Then, using the fact that the incident wave is a plane wave along the $z$ axis, i.e., $\mathbf{E}^{\mathrm{inc}}=\left(E_{x}, E_{y}, 0\right) e^{i k_{0} z}$ and $\mathbf{H}^{\mathrm{inc}}=\left(H_{x}, H_{y}, 0\right) e^{i k_{0} z}$, the force along the $x$ axis for two identical spheres can be written as

$$
\begin{aligned}
F_{x}\left(\mathbf{r}_{1}\right)= & \frac{1}{2} \operatorname{Re}\left\{\partial_{x} T_{x x}^{\mathrm{ee}}\left(\mathbf{r}_{1}, \mathbf{r}_{2}\right) \|\left.\alpha^{\mathrm{e}} E_{x}\right|^{2}+\partial_{x} T_{y y}^{\mathrm{ee}}\left(\mathbf{r}_{1}, \mathbf{r}_{2}\right)\left|\alpha^{\mathrm{e}} E_{y}\right|^{2}\right. \\
& +\partial_{x} T_{y y}^{\mathrm{hh}}\left(\mathbf{r}_{1}, \mathbf{r}_{2}\right)\left|\alpha^{\mathrm{m}} H_{y}\right|^{2}+\partial_{x} T_{x x}^{\mathrm{hh}}\left(\mathbf{r}_{1}, \mathbf{r}_{2}\right)\left|\alpha^{\mathrm{m}} H_{x}\right|^{2} \\
& -\frac{2}{3} k_{0}^{4}\left[-\alpha^{\mathrm{e}} T_{z y}^{\mathrm{eh}}\left(\mathbf{r}_{1}, \mathbf{r}_{2}\right)\left|\alpha^{\mathrm{m}} H_{y}\right|^{2}\right. \\
& \left.\left.+\left[\alpha^{\mathrm{m}} T_{z y}^{\mathrm{he}}\left(\mathbf{r}_{1}, \mathbf{r}_{2}\right)\right]^{*}\left|\alpha^{\mathrm{e}} E_{y}\right|^{2}\right]\right\},
\end{aligned}
$$

where $\partial_{u}$ denotes partial differentiation with respect to component $u$ of $\mathbf{r}_{1}$ (for the general expression, i.e., with two spheres with different material parameters, see Appendix A). The expressions of the field susceptibility tensors used in this paper are provided in Appendix B. Using the symmetry properties of the field susceptibility tensor, i.e., $\mathbf{T}^{\mathrm{ee}}=\mathbf{T}^{\mathrm{hh}}, \mathbf{T}^{\mathrm{eh}}=-\mathbf{T}^{\mathrm{he}}$, and $T_{x x}=T_{y y}$ and the properties of the incident field, i.e., $\mathbf{H}^{\text {inc }}=\left(-E_{y}, E_{x}, 0\right) e^{i k_{0} z}$, Eq. (7) can be simplified. Moreover if, as it is the case in this section, we assume that $\varepsilon=\mu$, i.e., $\alpha^{\mathrm{e}}=\alpha^{\mathrm{m}}=\alpha$, Eq. (7) can be further simplified to read as

$$
\begin{aligned}
F_{x}\left(\mathbf{r}_{1}\right)= & |\alpha|^{2} \operatorname{Re}\left\{\partial_{x} T_{x x}^{\mathrm{ee}}\left(\mathbf{r}_{1}, \mathbf{r}_{2}\right)+\partial_{x} T_{y y}^{\mathrm{ee}}\left(\mathbf{r}_{1}, \mathbf{r}_{2}\right)\right. \\
& \left.+\frac{1}{3} k_{0}^{4} \alpha T_{z y}^{\mathrm{eh}}\left(\mathbf{r}_{1}, \mathbf{r}_{2}\right)\right\}\left(\left|E_{x}\right|^{2}+\left|E_{y}\right|^{2}\right) .
\end{aligned}
$$

Notice that, as the spheres are small compared to the wavelength of illumination, the $k_{0}^{4} \alpha T$ term is negligible compared to the two other terms. If the electric field is linearly polarized and $\theta$ is the angle that the field makes with the positive $x$ direction, i.e., $\mathbf{E}^{\text {inc }}=E^{\text {inc }}(\cos \theta, \sin \theta, 0) e^{i k_{0} z}$, it is clear from Eq. (8) that the force experienced by sphere 1 is independent of $\theta$, hence the force along the $x$ axis is the same irrespective of the orientation of the electric field of the incident wave. Note that this behavior is different from what one would observe with two identical dielectric particles as shown in Fig. 2 and discussed in Ref. 26.

Having derived the general expression of the optical force, we now examine the long- and short-range behaviors of the force. Using Eq. (7), the far-field approximation for the force on sphere 1 for the $x$ polarization reads as

$$
\begin{aligned}
F_{x}\left(\mathbf{r}_{1}\right) & \approx \mathbf{u} \operatorname{Re}\left[\frac{k_{0}^{2}}{a^{2}} e^{i k_{0} a}\left|\alpha^{\mathrm{e}} E^{\mathrm{inc}}\right|^{2}+i \frac{k_{0}^{3}}{2 a} e^{i k_{0} a}\left|\alpha^{\mathrm{m}} H^{\mathrm{inc}}\right|^{2}\right] \\
& \approx-\frac{k_{0}^{3}}{2 a} \mathbf{u} \sin \left(k_{0} a\right)\left|\alpha^{\mathrm{m}} H^{\mathrm{inc}}\right|^{2} .
\end{aligned}
$$

Equation (9) shows that the far-field oscillations of the force are mainly due to the magnetic field when the incident electric field is oriented along the $x$ direction. As shown in Fig. 2(b), this is the same far-field behavior that would be observed between two identical dielectric spheres when the electric incident field is along the $y$ axis. In the short range (near field), the force on sphere 1 reads as

$$
\begin{aligned}
F_{x}\left(\mathbf{r}_{1}\right) & \approx \frac{\mathbf{u}}{2} \operatorname{Re}\left[-\frac{6}{a^{4}}\left|\alpha^{\mathrm{e}} E^{\mathrm{inc}}\right|^{2}+\frac{3}{a^{4}}\left|\alpha^{\mathrm{m}} H^{\mathrm{inc}}\right|^{2}\right] \\
& \approx-\frac{3 \mathbf{u}}{2 a^{4}}\left|\alpha E^{\mathrm{inc}}\right|^{2} .
\end{aligned}
$$

This time, the spatial dependence of the force is similar to that of the force between two identical dielectric spheres when the electric incident field is along the $x$ axis, one difference being that the magnitudes of the force differ by a factor of 2 as shown in Fig. 2(a).

It can also be shown that the $y$ component of the force is always zero, irrespective of the orientation of the incident field and the distance between the two spheres. This can be shown from Eq. (A4) but the result is more general and holds even if no approximation is made, i.e., using Eq. (A2). A consequence of this result for the dynamics of the system is that the spheres can not orbit around their common center of mass irrespective of the orientation of the incident field. This is unlike the case of two identical dielectric spheres where a torque arises if $\theta$ is different from $0^{\circ}$ or $90^{\circ} .^{28}$ 


\section{Illumination with a circular polarization}

We now consider the case of a circularly polarized incident wave, i.e., $\mathbf{E}^{\text {inc }}=\frac{E^{\text {inc }}}{\sqrt{2}}(1, i, 0) e^{i k_{0} z}$. Whereas the spatial dependence of the $x$ component of the force [Fig. 3(a)] is the same as for two identical dielectric spheres (up to an overall factor of 2 because $\mu=\varepsilon=2$ ), the $y$ component of the force [Fig. 3(b)] behaves differently in the MD case. First, its magnitude is not simply twice the that of the force for the nonmagnetic case. Second, the spatial period of the oscillations is also different from the nonmagnetic case. To better understand these differences, an analytical expression for the $y$ component of the optical force would be useful. However, one should proceed cautiously in deriving such an expression because if we use the approximation $T \alpha \ll 1$ we introduced previously, i.e., if we use Eq. (A4), we find a zero $y$ component of the force while the DDA computation predicts a nonzero value. This means that the optical force should be computed not with the approximation $T \alpha \ll 1$, but from the exact analytical expression of Eq. (A2). Moreover, for our configuration, by symmetry we have $E_{x}\left(\mathbf{r}_{1}\right)=E_{x}\left(\mathbf{r}_{2}\right), E_{y}\left(\mathbf{r}_{1}\right)=E_{y}\left(\mathbf{r}_{2}\right)$, $H_{x}\left(\mathbf{r}_{1}\right)=H_{x}\left(\mathbf{r}_{2}\right), H_{y}\left(\mathbf{r}_{1}\right)=H_{y}\left(\mathbf{r}_{2}\right)$ and $E_{z}\left(\mathbf{r}_{1}\right)=-E_{z}\left(\mathbf{r}_{2}\right)$, $H_{z}\left(\mathbf{r}_{1}\right)=-H_{z}\left(\mathbf{r}_{2}\right)$, from which Eq. (A2) can be written as

$$
\begin{aligned}
F_{y}\left(\mathbf{r}_{1}\right)= & \frac{1}{2} \operatorname{Re}\left\{2\left(\partial_{y} T_{x y}^{\mathrm{ee}}\right)^{*}\left\{\left|\alpha^{\mathrm{e}}\right|^{2} \operatorname{Re}\left[E_{x}\left(\mathbf{r}_{1}\right) E_{y}^{*}\left(\mathbf{r}_{1}\right)\right]+\left|\alpha^{\mathrm{m}}\right|^{2} \operatorname{Re}\left[H_{x}\left(\mathbf{r}_{1}\right) H_{y}^{*}\left(\mathbf{r}_{1}\right)\right]\right\}\right. \\
& \left.-2\left(\partial_{y} T_{x z}^{\mathrm{he}}\right)^{*} \operatorname{Re}\left\{i \alpha^{\mathrm{e}}\left(\alpha^{\mathrm{m}}\right)^{*}\left[E_{x}\left(\mathbf{r}_{1}\right) H_{z}^{*}\left(\mathbf{r}_{1}\right)+E_{z}\left(\mathbf{r}_{1}\right) H_{x}^{*}\left(\mathbf{r}_{1}\right)\right]\right\}-\frac{2}{3} k_{0}^{4}\left\{\alpha^{\mathrm{e}}\left(\alpha^{\mathrm{m}}\right)^{*}\left[-E_{x}\left(\mathbf{r}_{1}\right) H_{z}^{*}\left(\mathbf{r}_{1}\right)+E_{z}\left(\mathbf{r}_{1}\right) H_{x}^{*}\left(\mathbf{r}_{1}\right)\right]\right\}\right\} .
\end{aligned}
$$

In the case where $\alpha^{\mathrm{e}}=\alpha^{\mathrm{m}}=\alpha$ (two identical MD particles with $\varepsilon=\mu$ ), the first two terms cancel out and we get

$$
F_{y}\left(\mathbf{r}_{1}\right)=-|\alpha|^{2} \operatorname{Re}\left\{\left(\partial_{y} T_{x z}^{\mathrm{he}}\right)^{*} \operatorname{Re}\left\{i\left[E_{x}\left(\mathbf{r}_{1}\right) H_{z}^{*}\left(\mathbf{r}_{1}\right)+E_{z}\left(\mathbf{r}_{1}\right) H_{x}^{*}\left(\mathbf{r}_{1}\right)\right]\right\}+\frac{1}{3} k_{0}^{4}\left\{\left[-E_{x}\left(\mathbf{r}_{1}\right) H_{z}^{*}\left(\mathbf{r}_{1}\right)+E_{z}\left(\mathbf{r}_{1}\right) H_{x}^{*}\left(\mathbf{r}_{1}\right)\right]\right\}\right\} .
$$

As a sanity check, we note that for a linearly polarized incident wave, the expressions in square brackets in Eq. (12) are imaginary which results in $F_{y}\left(\mathbf{r}_{1}\right)=0$, as discussed in the previous section. Also, from Eq. (12) we can check that, for a circularly polarized incident wave, in the case of two dielectrics spheres, i.e., Eq. (11) with $\alpha^{\mathrm{m}}=0$, the force in far field can be written as

$$
\begin{aligned}
F_{y}\left(\mathbf{r}_{1}\right)= & \left|\alpha^{\mathrm{e}}\right|^{2}\left|E_{0}\right|^{2} \frac{k_{0}^{4} \mathbf{u}}{a^{3}} \\
& \times\left[\operatorname{Re}\left(\alpha^{\mathrm{e}}\right) \frac{\sin \left(2 k_{0} a\right)}{2}+\operatorname{Im}\left(\alpha^{\mathrm{e}}\right) \cos ^{2}\left(k_{0} a\right)\right] .
\end{aligned}
$$

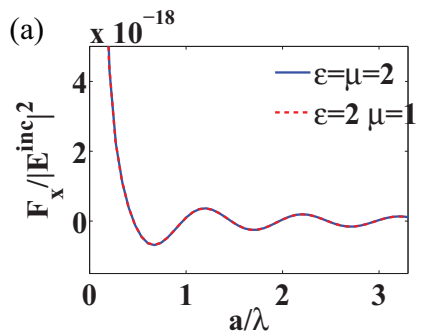

(c)
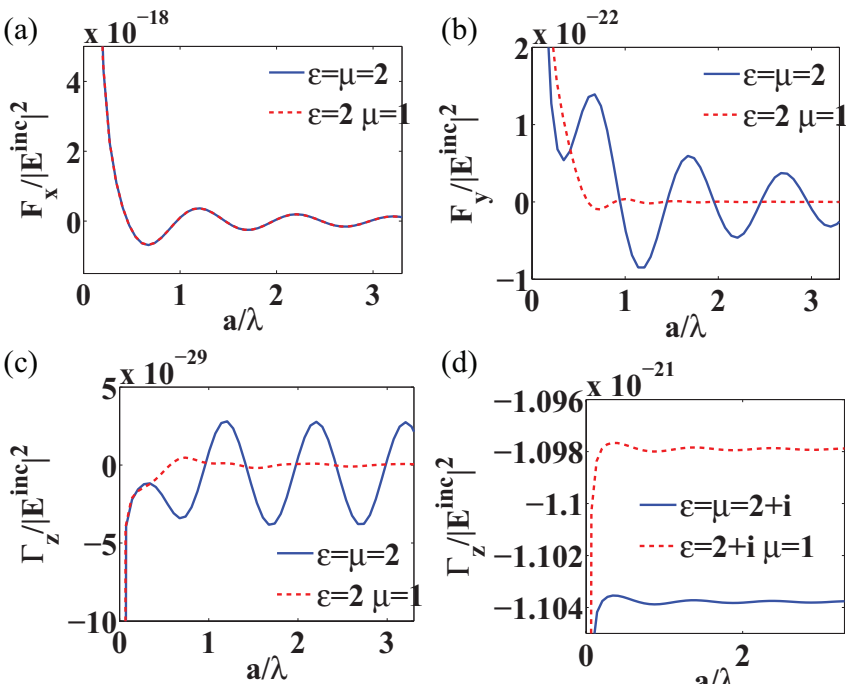

(d)

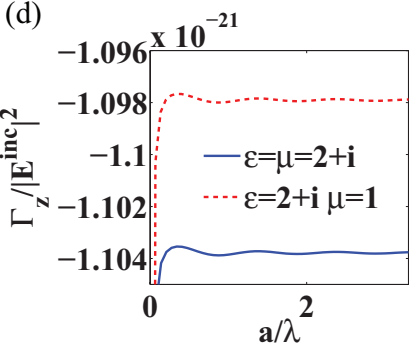

FIG. 3. (Color online) Force and torque on sphere 1 for a system of two spheres with radius of $r=\lambda / 60$. The incident plane wave is circularly polarized. Dashed lines: identical dielectric spheres $(\varepsilon=2$, $\mu=1)$; solid lines: identical magnetodielectric spheres $(\varepsilon=\mu=2)$. (a) $x$ component of the force; (b) $x$ component of the force; (c) $x$ component of the torque; (d) same as (c) but with an absorbing part added to the permittivity or permeability.
If the particles are lossless, we recover the result derived in Ref. 28, i.e., $F_{y}$ oscillates with the separation between the spheres with a period of oscillation of $\lambda / 2$, and an amplitude envelope that falls off as $1 / a^{3}$.

The far-field behavior of the $y$ component of the force for MD spheres is dramatically different from the nonmagnetic case. This difference comes from the second term of Eq. (12) which varies as $1 / a$; the first term falls off faster with the separation (as $1 / a^{2}$ ) due to its dependence on $\partial_{y} T_{x z}^{\text {he }}$ (notice that as emphasized in Ref. 53, the second term in the case of dipole approximation is not negligible). By using the farfield approximation $E_{x}\left(\mathbf{r}_{1}\right) \approx E_{x}, H_{x}\left(\mathbf{r}_{1}\right) \approx H_{x}$ and $E_{z}\left(\mathbf{r}_{1}\right)=$ $-\alpha T_{y z}^{\mathrm{eh}} H_{y}, H_{z}\left(\mathbf{r}_{1}\right)=\alpha T_{y z}^{\mathrm{eh}} E_{y}$, the $y$ component of the force in the far field can be finally expressed as

$$
\begin{aligned}
F_{y}\left(\mathbf{r}_{1}\right)= & -\frac{1}{3}|\alpha|^{2}\left|E^{\mathrm{inc}}\right|^{2} \frac{k_{0}^{6} \mathbf{u}}{a} \\
& \times\left[\operatorname{Re}(\alpha) \sin \left(k_{0} a\right)+\operatorname{Im}(\alpha) \cos \left(k_{0} a\right)\right] .
\end{aligned}
$$

As we have $F_{y}\left(\mathbf{r}_{2}\right)=-F_{y}\left(\mathbf{r}_{1}\right)$, the torque in the far field is given by

$$
\begin{aligned}
\Gamma_{z}\left(\mathbf{r}_{1}\right)= & \Gamma_{z}\left(\mathbf{r}_{2}\right) \\
= & -\frac{1}{3}|\alpha|^{2}\left|E^{\operatorname{inc}}\right|^{2} k_{0}^{6}\left[\operatorname{Re}(\alpha) \sin \left(k_{0} a\right)\right. \\
& \left.+\operatorname{Im}(\alpha) \cos \left(k_{0} a\right)\right] .
\end{aligned}
$$

As shown by Eq. (15), a consequence of the $1 / a$ spatial dependence of the $y$ component of the optical force is that the magnitude (or more specifically its envelope) of the optical torque of the nanomixer formed by both spheres does not weaken with the separation $a$ between the spheres. This is illustrated in Fig. 3(c). This peculiar behavior of the torque results from the $1 / a$ dependence of the $y$ component of the force. Let us take a closer look at this configuration. For nonmagnetic Rayleigh particles, the optical force is commonly described in terms of the gradient force, which tends to draw the particles toward, say, the high-intensity regions (this depends on the polarizability of the particles), or radiation pressure which tends to "push" the particle along in the 
direction of the Poynting vector associated with the incident field. In the present case, however, these usual pictures clearly fail to capture the physics of the problem. As it happens, the unusual torque is due to a magnetodielectric effect. Indeed, for a magnetodielectric particle illuminated by an electromagnetic wave, the requirement that the total energy of the system is conserved yields a "recoil" force for the particle. ${ }^{54,55}$ This recoil force originates from the coupling between the induced electric and magnetic dipole moments in the particle. The direction of the force is normal to the plane formed by the two induced dipole moments.

In our case, we must keep in mind two things. First, because we have magnetodielectric particles, the electric and the magnetic fields of the incident wave will each induce a pair of dipole moments (one electric and one magnetic) in each particle. Second, because we are considering isotropic materials, the (transverse) incident field can not induce a dipole moment in the $z$ direction. However, each particle is also exposed to the field scattered by the other particle. This secondary, scattered field can induce dipole moments in the $z$ direction. Once the direct (incident field) and indirect (field due to the other particle) polarization contributions are taken into account, the coupling between the electric and magnetic dipole moments of each particle (e.g., electric and magnetic dipole moment along $x$ due to the incident field, and magnetic and electric dipole moment along $z$ due to the scattered field) results in a recoil force perpendicular to the $(x, z)$ plane and hence along $y$.

Regarding the spatial dependence of the force, for a given particle, the force along $y$ stems for the interaction of a dipole moment induced by the incident field (which has constant envelope) and the far-field radiated by the dipole moment of the other particle (whose envelope decays as $1 / a$ since we are in far field). Hence, the net spatial dependence of the force is $1 / a$. If the spheres are lossless, the optical torque and the optical force can be written as

$$
\begin{aligned}
& \Gamma_{z}\left(\mathbf{r}_{1}\right)=-\frac{1}{3}|\alpha|^{2}\left|E^{\text {inc }}\right|^{2} k_{0}^{6} \alpha \sin \left(k_{0} a\right), \\
& F_{x}\left(\mathbf{r}_{1}\right)=-\frac{k_{0}^{3}}{2 a} \mathbf{u}\left|\alpha E^{\text {inc }}\right|^{2} \sin \left(k_{0} a\right) .
\end{aligned}
$$

We obtain the same oscillatory behavior for the $x$ component of the optical force and the $z$ component of the optical torque, i.e., a $\sin \left(k_{0} a\right)$ spatial dependence. Incidentally, from a dynamical point of view, this implies that when the separation between the particles is such that $F_{x}=0$, the optical torque also vanishes. Therefore, the spheres can not rotate around their common center of mass in stationary orbits. By contrast, if we introduce material absorption, writing the polarizabilities as $\alpha=|\alpha| e^{i \varphi}$, the $z$ component of the optical torque becomes

$$
\Gamma_{z}\left(\mathbf{r}_{1}\right)=-\frac{1}{3}|\alpha|^{3}\left|E^{\mathrm{inc}}\right|^{2} k_{0}^{6} \sin \left(k_{0} a+\varphi\right)
$$

and the expression of the optical force in Eq. (14) remains unchanged. Then, by choosing $\varphi=\pi / 2$, one gets a maximum of optical torque for a position of equilibrium which would favor the emergence of stable orbits.

Notice also that in the case of lossless particles, there is no intrinsic part for the optical torque as underlined in Refs. 40, 41 , and 43 [the second term of Eq. (6) vanishes], but this is no longer the case for an absorbing particle. In Fig. 3(d), an absorbing part is added to the relative permittivity/permeability such that $\varepsilon=\mu=2+i$ then we observed the same behavior as in Fig. 3(c), oscillation with a period of $\lambda$ but the oscillations are around the value of the intrinsic optical torque of the isolated sphere. The magnitude of the oscillations is increased as the modulus of the polarizability is stronger due to the absorbing part of $(\varepsilon, \mu)$.

\section{B. Symmetric case: Identical particles with $\varepsilon \neq \mu$}

The results of the previous section rely on having $\epsilon=\mu$. However, it is interesting to examine whether the long-range behavior of the torque is affected by breaking the symmetry between $\epsilon$ and $\mu$ (note that we are still referring to this situation as the "symmetric case" since the particles are identical).

\section{Illumination with linear polarization}

Assuming $k_{0}^{3} \alpha \ll 1$, from Eq. (7) for the linearly polarized field we get

$$
\begin{aligned}
F_{x}\left(\mathbf{r}_{1}\right)= & \left|E^{\mathrm{inc}}\right|^{2} \operatorname{Re}\left\{\left|\alpha^{\mathrm{e}}\right|^{2}\left[\partial_{x} T_{x x}^{\mathrm{ee}}\left(\mathbf{r}_{1}, \mathbf{r}_{2}\right)+\partial_{x} T_{y y}^{\mathrm{ee}}\left(\mathbf{r}_{1}, \mathbf{r}_{2}\right)\right]\right. \\
& +\left(\left|\alpha^{\mathrm{m}}\right|^{2}-\left|\alpha^{\mathrm{e}}\right|^{2}\right)\left[\partial_{x} T_{x x}^{\mathrm{ee}}\left(\mathbf{r}_{1}, \mathbf{r}_{2}\right) \sin ^{2} \theta\right. \\
& \left.\left.+\partial_{x} T_{y y}^{\mathrm{ee}}\left(\mathbf{r}_{1}, \mathbf{r}_{2}\right) \cos ^{2} \theta\right]\right\} .
\end{aligned}
$$

Clearly, having $\mu \neq \varepsilon$ induces a dependence of the force on the orientation of the electric field, similar to what we would get in the dielectric (i.e., nonmagnetic) case. For the $y$ component of the force, one can show that

$$
F_{y}\left(\mathbf{r}_{1}\right) \propto\left|E^{\text {inc }}\right|^{2} \sin \theta \cos \theta\left(\left|\alpha^{\mathrm{e}}\right|^{2}-\left|\alpha^{\mathrm{m}}\right|^{2}\right) .
$$

Hence, breaking the symmetry between $\varepsilon$ and $\mu$ results in an optical torque with a $\sin \theta \cos \theta$ spatial (angular) dependence, similar to the dielectric case.

\section{Illumination with circular polarization}

Assuming once again that $k_{0}^{3} \alpha \ll 1$, the $x$ component of the force reads as

$$
\begin{aligned}
F_{x}\left(\mathbf{r}_{1}\right)= & \frac{\left|E^{\mathrm{inc}}\right|^{2}}{4}\left(\left|\alpha^{\mathrm{e}}\right|^{2}+\left|\alpha^{\mathrm{m}}\right|^{2}\right) \\
& \times \operatorname{Re}\left\{\partial_{x} T_{x x}^{\mathrm{ee}}\left(\mathbf{r}_{1}, \mathbf{r}_{2}\right)+\partial_{x} T_{y y}^{\mathrm{ee}}\left(\mathbf{r}_{1}, \mathbf{r}_{2}\right)\right\} .
\end{aligned}
$$

This shows that the spatial behavior of the optical binding force is independent of the values of $\varepsilon$ and $\mu$. Unlike in the previous section, for the $y$ component of the force, which is responsible for the optical torque along the $z$ axis, the first two terms of Eq. (11) do not cancel out. This yields an expression similar to Eq. (13) with a force that decays as $1 / a^{3}$ in far field. Therefore, only the term due to the coupling between the electric and magnetic dipoles remains in the far field:

$$
F_{y}\left(\mathbf{r}_{1}\right)=-\frac{1}{6} k_{0}^{4}\left|E^{\mathrm{inc}}\right|^{2} \operatorname{Re}\left\{i \alpha^{e}\left(\alpha^{m}\right)^{*}\left[\left(T_{x z}^{\mathrm{eh}} \alpha^{e}\right)^{*}-T_{x z}^{\mathrm{eh}} \alpha^{m}\right]\right\} .
$$

Due to the $1 / a$ asymptotic behavior of $T_{x z}^{\mathrm{eh}}, F_{y}$ also decays as $1 / a$ with the separation between the particles. As a result, the envelope of the optical torque does not decay with the distance between the particles. In other words, a small (i.e., $\mu \ll \varepsilon$ ) magnetic response in the particles is enough to yield an asymptotically nondecreasing optical torque. Equality between $\varepsilon$ and $\mu$ is not required. However, the closer $\epsilon$ and $\mu$, 

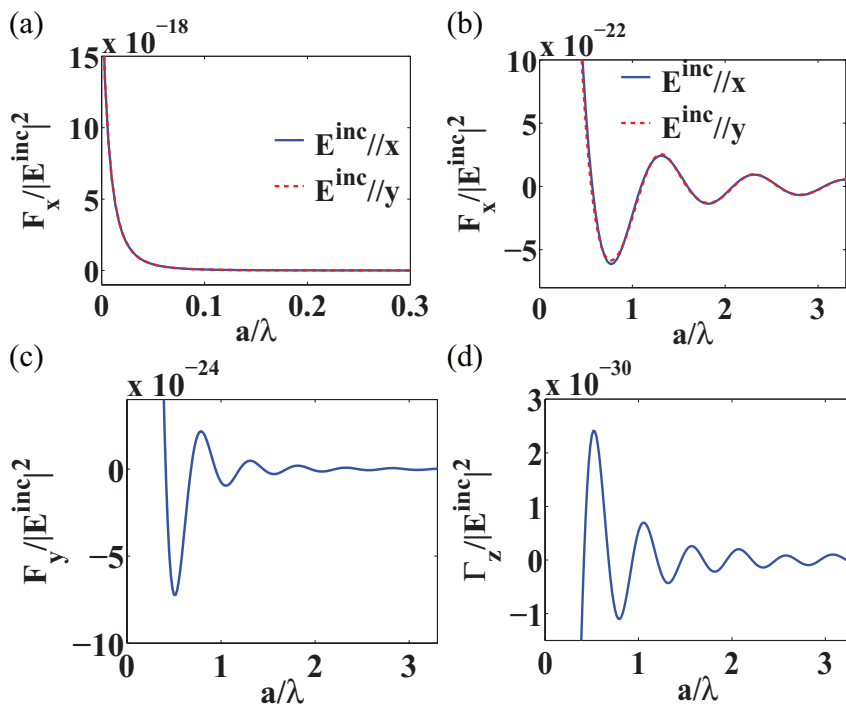

(d)

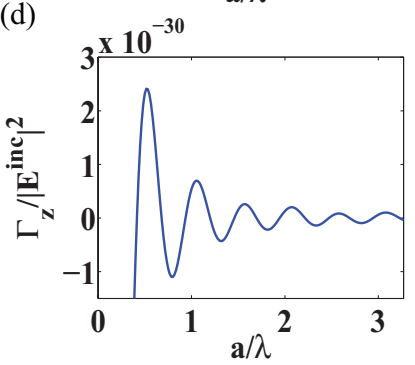

FIG. 4. (Color online) Study when sphere 1 is dielectric $\left(\varepsilon_{1}=2\right.$, $\left.\mu_{1}=1\right)$ and sphere 2 magnetic $\left(\varepsilon_{1}=1, \mu_{1}=2\right)$. $x$ component of the optical force experiences by the sphere 1 versus $x / \lambda$ in near field (a) and far field (b) when both spheres are illuminated with a linear polarization. (c) $y$ component of the optical force and (a) $z$ component of the optical torque experience by sphere 1 when illuminated with a circular polarization.

the faster the envelope of the torque will stop oscillating as the distance between the particles increases.

\section{Antisymmetric case: One dielectric and one magnetic sphere}

In this section, both particles have the same radius $r=\lambda / 60$ but sphere 1 is purely dielectric $\left(\mu_{1}=1\right)$ and sphere 2 is purely magnetic with $\left(\varepsilon_{2}=1\right)$.

\section{Illumination with linear polarization}

We start by examining the validity of the dipole approximation for this configuration. We plot the $x$ component of the optical force versus $a / \lambda$, in the near-field [Fig. 4(a)] and far-field [Fig. 4(b)] regimes. In the dipole approximation, the $x$ component of the force on sphere 1 is

$$
\begin{aligned}
F_{x}\left(\mathbf{r}_{1}\right)= & \frac{1}{2} \operatorname{Re}\left[-\left|\alpha^{\mathrm{e}} E_{y}\right|^{2}\left(\alpha^{\mathrm{m}} T_{y z}^{\mathrm{eh}} \partial_{x} T_{y z}^{\mathrm{eh}}\right)^{*}\right. \\
& \left.+\left|\alpha^{\mathrm{m}} H_{y}\right|^{2} \alpha^{\mathrm{e}} T_{y z}^{\mathrm{eh}}\left(\partial_{x} T_{y z}^{\mathrm{eh}}\right)^{*}\right] .
\end{aligned}
$$

In the case of $\alpha^{\mathrm{e}}=\alpha^{\mathrm{m}}=\alpha$, i.e., $\varepsilon_{1}=\mu_{2}$, the near-field and far-field approximations of the optical force can be written as

$$
\begin{gathered}
F_{x}^{\text {near }}\left(\mathbf{r}_{1}\right)=\frac{\mathbf{u}}{a^{5}} k_{0}^{2} \operatorname{Re}(\alpha)\left[\left|\alpha E_{y}\right|^{2}+\left|\alpha H_{y}\right|^{2}\right], \\
F_{x}^{\mathrm{far}}\left(\mathbf{r}_{1}\right)=-\frac{k_{0}^{5} \mathbf{u}}{2 a^{2}} \operatorname{Re}\left[\left|\alpha E_{y}\right|^{2}\left(\alpha i e^{2 i k_{0} a}\right)^{*}+i\left|\alpha H_{y}\right|^{2} \alpha\right] .
\end{gathered}
$$

Hence, in the near-field regime, we observe a strong decrease of the optical binding when the two spheres are illuminated with linear polarization, irrespective of the orientation of the electric field [from Eq. (23), we obviously get $F_{x}\left(\mathbf{r}_{2}\right)=$ $\left.-F_{x}\left(\mathbf{r}_{1}\right)\right]$. In the far field, the dipole approximation (24) predicts that for an incident field linearly polarized along the $x$ axis, the optical force on sphere 1 varies largely monotonically with the distance between both spheres, whereas sphere 2 experiences an oscillating optical force with period $\lambda / 2$.

However, despite the fact that the spheres are small compared to the wavelength, this picture is incorrect because the dipole approximation fails. Figure 4(b) shows that the full computation (DDA) predicts that both spheres will experience the same force (opposite in sign), the magnitude of which oscillates with period $\lambda$, as the distance between the spheres increases. In fact, in this particular case, the dipole approximation fails because the spatial variation of the fields inside the sphere is not negligible. In other words, the electric dipole associated with sphere 1 interacts too strongly with the magnetic quadrupole associated with sphere 2 for the quadrupolar contribution to be neglected. If we take the quadrupolar order into account, ${ }^{56,57}$ we obtain the following, polarization-independent, far-field approximation for the optical binding (see Appendix C for more details):

$$
F_{x}\left(\mathbf{r}_{1}\right)=\frac{k_{0}^{4} \mathbf{u}}{18 a^{2}} \operatorname{Re}\left[\alpha^{\mathrm{e}}\left(\alpha_{\mathrm{q}}^{\mathrm{m}} e^{i k_{0} a}\right)^{*}\right]\left|E^{\mathrm{inc}}\right|^{2},
$$

where $\alpha_{\mathrm{q}}^{\mathrm{m}}$ is the quadrupolar magnetic polarizability of sphere 2. As the dipolar polarizability is proportional to $r^{3}$ ( $r$ being the radius of the sphere) and the quadrupolar polarizability is proportional to $r^{5}$, the overall force is proportional to $r^{8}$, whereas the corresponding dipole approximation (24) predicts a force proportional to $r^{9}$. Hence, the spatial dependence of the dipole-quadrupole coupling is one order of magnitude larger than the dipole-dipole coupling.

The $y$ component of the optical force, on the other hand, is accurately described within the dipole approximation

$$
\begin{aligned}
F_{y}\left(\mathbf{r}_{1}\right)= & \frac{|\alpha|^{2}}{2} \operatorname{Re}\left\{\left[\alpha T_{y z}^{\mathrm{eh}}\left(\mathbf{r}_{1}, \mathbf{r}_{2}\right) \partial_{y} T_{x z}^{\mathrm{eh}}\left(\mathbf{r}_{1}, \mathbf{r}_{2}\right)\right]^{*} E_{x} E_{y}^{*}\right. \\
& \left.-\left\{\alpha T_{y z}^{\mathrm{eh}}\left(\mathbf{r}_{1}, \mathbf{r}_{2}\right)\left[\partial_{y} T_{x z}^{\mathrm{eh}}\left(\mathbf{r}_{1}, \mathbf{r}_{2}\right)\right]^{*}\right\} H_{y} H_{x}^{*}\right\}, \\
F_{y}\left(\mathbf{r}_{2}\right)= & \frac{|\alpha|^{2}}{2} \operatorname{Re}\left\{-\left[\alpha T_{y z}^{\mathrm{eh}}\left(\mathbf{r}_{1}, \mathbf{r}_{2}\right) \partial_{y} T_{x z}^{\mathrm{eh}}\left(\mathbf{r}_{1}, \mathbf{r}_{2}\right)\right]^{*} H_{x} H_{y}^{*}\right. \\
& \left.+\left\{\alpha T_{y z}^{\mathrm{eh}}\left(\mathbf{r}_{1}, \mathbf{r}_{2}\right)\left[\partial_{y} T_{x z}^{\mathrm{eh}}\left(\mathbf{r}_{1}, \mathbf{r}_{2}\right)\right]^{*}\right\} E_{y} E_{x}^{*}\right\} .
\end{aligned}
$$

From this we can deduce that when the linearly polarized incident electric field has nonzero components on both the $x$ and $y$ axes, the force has a nonzero component in the $y$ direction. However, we also have $F_{y}\left(\mathbf{r}_{1}\right)=F_{y}\left(\mathbf{r}_{2}\right)$ which means that the two spheres are pushed along by the incident field without experiencing any torque. This behavior is contrary to that which would be observed for two identical dielectric spheres, as in that case the $y$ components of the optical force at the spheres are of opposite sign. The associated optical torque would align the system of two spheres on the direction of the incident electric field. This is akin to an ellipsoidal particles which would have a tendency to align itself along its long or short axes, although stable equilibrium is only achieved along the long axis. ${ }^{40,58}$

\section{Illumination with circular polarization}

For circular polarization, the $x$ component of the optical force is identical to the one found for linear polarization. This is obvious from Eq. (25) as $F_{x}$ depends only on the modulus of the electric field. The $y$ components of the force at the two spheres are opposite in sign, which can yield a nonzero 
optical torque. If we derive the far-field expressions for the $y$ component of the optical force and the $z$ component of the optical torque, we obtain

$$
\begin{gathered}
F_{y}\left(\mathbf{r}_{1}\right)=-F_{y}\left(\mathbf{r}_{2}\right) \\
=-|\alpha|^{2} \frac{\mathbf{u}}{a^{3}} k_{0}^{4}\left[\operatorname{Re}(\alpha) \sin \left(2 k_{0} a\right)\right. \\
\left.-\operatorname{Im}(\alpha) \sin ^{2}\left(k_{0} a\right)\right], \\
\Gamma_{z}=|\alpha|^{2} \frac{k_{0}^{4}}{a^{2}}\left[\operatorname{Re}(\alpha) \sin \left(2 k_{0} a\right)-\operatorname{Im}(\alpha) \sin ^{2}\left(k_{0} a\right)\right] .
\end{gathered}
$$

The period of the oscillations for both the torque $\Gamma_{z}$ and the force $F_{y}$ is $\lambda / 2$, as seen in Figs. 4(c) and 4(d). We also note the rapid decay of the force $F_{y}$ which falls off as $1 / a^{3}$. By contrast, note that in this configuration the period of the $x$ component of the force is $\lambda$.

\section{CONCLUSION}

We have studied the optical binding and optical torque between two magnetodielectric particles. We found that the period of oscillations and the damping of the force and torque strongly depends of the illumination and the nature of the particles. In Table I, we summarize our results for lossless particles.

In the far field, the period of the oscillations of the force is always $\lambda$, irrespective of the illumination and the nature of the particles. For a system of two MD particles, however, the force and the torque have a different spatial dependence. The properties of the torque are also qualitatively different from the case of two dielectric particles as, for two MD particles, the optical torque is always zero under linear illumination. For circular illumination, two cases must be distinguished. For
TABLE I. Period of oscillations and decay with distance in far field for the optical force and torque, in the case of a lossless, magnetodielectric Rayleigh particle.

\begin{tabular}{llccccc}
\hline \hline & & \multicolumn{2}{c}{ Force } & & \multicolumn{2}{c}{ Torque } \\
\cline { 3 - 4 } Case & Polarization & Period & Decay & & Period & Decay \\
\hline \multirow{3}{*}{ Antisymmetric } & Circular & $\lambda$ & $1 / a$ & & $\lambda$ & 1 \\
& Linear & $\lambda$ & $1 / a^{2}$ & & \\
& Circular & $\lambda$ & $1 / a^{2}$ & & $\lambda / 2$ & $1 / a^{2}$ \\
\hline \hline
\end{tabular}

a symmetric particle configuration, the system experiences an optical torque which oscillates with spatial period $\lambda$ as the distance between the particles increases. However, the magnitude of the optical torque is independent of the distance between the two particles. For an antisymmetric configuration, on the other hand, the long-range component of the force oscillates with a period $\lambda / 2$ and both the force and the torque decay at the same rate when the separation between the particles increases. Now that the canonical case of the optical force and torque on two Rayleigh particles has been extended to MD particles, the next step would be to study optical binding for MD particles large compared to the wavelength of illumination, and see how size effects will affect the force and torques, particularly regarding the undamped optical torque of the symmetric case. Once the size effect is determined, an interesting question is whether bound (stable) states can be created for a system of more than two particles.

\section{ACKNOWLEDGMENT}

A. Rahmani acknowledges the hospitality and support of the Ecole Centrale de Marseille during part of this work.

\section{APPENDIX A: OPTICAL FORCES BETWEEN TWO DIFFERENT SPHERES}

In this Appendix, we give the full expressions for the component of the optical force, without any approximation, using only $\left\{\mathbf{E}\left(\mathbf{r}_{1}\right), \mathbf{H}\left(\mathbf{r}_{1}\right)\right\}$ and $\left\{\mathbf{E}\left(\mathbf{r}_{2}\right), \mathbf{H}\left(\mathbf{r}_{2}\right)\right\}$, the electromagnetic local fields at the spheres, respectively (i.e., without the derivative of the local field). The force along the $x$ direction between the two spheres can be written as

$$
\begin{aligned}
F_{x}\left(\mathbf{r}_{1}\right)= & \frac{1}{2} \operatorname{Re}\left\{\left[\partial_{x} T_{x x}^{\mathrm{ee}}\left(\mathbf{r}_{1}, \mathbf{r}_{2}\right)\right]^{*}\left[\alpha^{\mathrm{e}}\left(\mathbf{r}_{2}\right)\right]^{*} \alpha^{\mathrm{e}}\left(\mathbf{r}_{1}\right) E_{x}\left(\mathbf{r}_{1}\right) E_{x}^{*}\left(\mathbf{r}_{2}\right)+\left[\partial_{x} T_{x x}^{\mathrm{hh}}\left(\mathbf{r}_{1}, \mathbf{r}_{2}\right)\right]^{*}\left[\alpha^{\mathrm{m}}\left(\mathbf{r}_{2}\right)\right]^{*} \alpha^{\mathrm{m}}\left(\mathbf{r}_{1}\right) H_{x}\left(\mathbf{r}_{1}\right) H_{x}^{*}\left(\mathbf{r}_{2}\right)\right. \\
& +\left[\partial_{x} T_{y y}^{\mathrm{ee}}\left(\mathbf{r}_{1}, \mathbf{r}_{2}\right)\right]^{*} x\left[\alpha^{\mathrm{e}}\left(\mathbf{r}_{2}\right)\right]^{*} \alpha^{\mathrm{e}}\left(\mathbf{r}_{1}\right)\left[E_{y}\left(\mathbf{r}_{1}\right) E_{y}^{*}\left(\mathbf{r}_{2}\right)+E_{z}\left(\mathbf{r}_{1}\right) E_{z}^{*}\left(\mathbf{r}_{2}\right)\right] \\
& +\left[\partial_{x} T_{y y}^{\mathrm{hh}}\left(\mathbf{r}_{1}, \mathbf{r}_{2}\right)\right]^{*}\left[\alpha^{\mathrm{m}}\left(\mathbf{r}_{2}\right)\right]^{*} \alpha^{\mathrm{m}}\left(\mathbf{r}_{1}\right)\left[H_{y}\left(\mathbf{r}_{1}\right) H_{y}^{*}\left(\mathbf{r}_{2}\right)+H_{z}\left(\mathbf{r}_{1}\right) H_{z}^{*}\left(\mathbf{r}_{2}\right)\right] \\
& +\left[\partial_{x} T_{y z}^{\mathrm{he}}\right]^{*}\left[\alpha^{\mathrm{e}}\left(\mathbf{r}_{1}\right)\left[\alpha^{\mathrm{m}}\left(\mathbf{r}_{2}\right)\right]^{*}\left[-E_{y}\left(\mathbf{r}_{1}\right) H_{z}^{*}\left(\mathbf{r}_{2}\right)+E_{z}\left(\mathbf{r}_{1}\right) H_{y}^{*}\left(\mathbf{r}_{2}\right)\right]+\alpha^{\mathrm{m}}\left(\mathbf{r}_{1}\right)\left[\alpha^{\mathrm{e}}\left(\mathbf{r}_{2}\right)\right]^{*}\left[H_{y}\left(\mathbf{r}_{1}\right) E_{z}^{*}\left(\mathbf{r}_{2}\right)-H_{z}\left(\mathbf{r}_{1}\right) E_{y}^{*}\left(\mathbf{r}_{2}\right)\right]\right] \\
& \left.-\frac{2}{3} k_{0}^{4} \alpha^{\mathrm{e}}\left(\mathbf{r}_{1}\right)\left[\alpha^{\mathrm{m}}\left(\mathbf{r}_{1}\right)\right]^{*}\left[E_{y}\left(\mathbf{r}_{1}\right) H_{z}^{*}\left(\mathbf{r}_{1}\right)-E_{z}\left(\mathbf{r}_{1}\right) H_{y}^{*}\left(\mathbf{r}_{1}\right)\right]\right\}
\end{aligned}
$$

and the $y$ component of the force can be written as

$$
\begin{aligned}
F_{y}\left(\mathbf{r}_{1}\right)= & \frac{1}{2} \operatorname{Re}\left\{\left[\partial_{y} T_{x y}^{\mathrm{ee}}\right]^{*}\left[\alpha^{\mathrm{e}}\left(\mathbf{r}_{2}\right)\right]^{*} \alpha^{\mathrm{e}}\left(\mathbf{r}_{1}\right)\left[E_{x}\left(\mathbf{r}_{1}\right) E_{y}^{*}\left(\mathbf{r}_{2}\right)+E_{y}\left(\mathbf{r}_{1}\right) E_{x}^{*}\left(\mathbf{r}_{2}\right)\right]\right. \\
& +\left[\partial_{y} T_{x y}^{\mathrm{hh}}\right]^{*}\left[\alpha^{\mathrm{m}}\left(\mathbf{r}_{2}\right)\right]^{*} \alpha^{\mathrm{m}}\left(\mathbf{r}_{1}\right)\left[H_{x}\left(\mathbf{r}_{1}\right) H_{y}^{*}\left(\mathbf{r}_{2}\right)+H_{y}\left(\mathbf{r}_{1}\right) H_{x}^{*}\left(\mathbf{r}_{2}\right)\right] \\
& +\left[\partial_{y} T_{x z}^{\mathrm{he}}\right]^{*}\left[\left[\alpha^{\mathrm{m}}\left(\mathbf{r}_{2}\right)\right]^{*} \alpha^{\mathrm{e}}\left(\mathbf{r}_{1}\right)\left[-E_{x}\left(\mathbf{r}_{1}\right) H_{z}^{*}\left(\mathbf{r}_{2}\right)+E_{z}\left(\mathbf{r}_{1}\right) H_{x}^{*}\left(\mathbf{r}_{2}\right)\right]\right. \\
& \left.+\left[\alpha^{\mathrm{e}}\left(\mathbf{r}_{2}\right)\right]^{*} \alpha^{\mathrm{m}}\left(\mathbf{r}_{1}\right)\left[H_{x}\left(\mathbf{r}_{1}\right) E_{z}^{*}\left(\mathbf{r}_{2}\right)-H_{z}\left(\mathbf{r}_{1}\right) E_{x}^{*}\left(\mathbf{r}_{2}\right)\right]\right] \\
& \left.-\frac{2}{3} k_{0}^{4} \alpha^{\mathrm{e}}\left(\mathbf{r}_{1}\right)\left[\alpha^{\mathrm{m}}\left(\mathbf{r}_{1}\right)\right]^{*}\left[-E_{x}\left(\mathbf{r}_{1}\right) H_{z}^{*}\left(\mathbf{r}_{1}\right)+E_{z}\left(\mathbf{r}_{1}\right) H_{x}^{*}\left(\mathbf{r}_{1}\right)\right]\right\} .
\end{aligned}
$$

Notice that in writing Eqs. (A1) and (A2) we have used the symmetry properties of the susceptibility tensors.

In the case where the approximation $\mathbf{T} \alpha \ll 1$ holds, and both spheres are illuminated with an incident electromagnetic plane wave $\mathbf{E}^{\text {inc }}=\left(E_{x}, E_{y}, 0\right) e^{i k_{0} z}$ and $\mathbf{H}^{\text {inc }}=\left(H_{x}, H_{y}, 0\right) e^{i k_{0} z}$, the optical force along the $x$ axis can be written as a function of the 
incident field as

$$
\begin{aligned}
F_{x}\left(\mathbf{r}_{1}\right)= & \frac{1}{2} \operatorname{Re}\left\{\left[\alpha^{\mathrm{e}}\left(\mathbf{r}_{2}\right)\right]^{*} \alpha^{\mathrm{e}}\left(\mathbf{r}_{1}\right)\left[\partial_{x} T_{x x}^{\mathrm{ee}}\left(\mathbf{r}_{1}, \mathbf{r}_{2}\right)\left|E_{x}\right|^{2}+\partial_{x} T_{y y}^{\mathrm{ee}}\left(\mathbf{r}_{1}, \mathbf{r}_{2}\right)\left|E_{y}\right|^{2}\right]^{*}\right. \\
& +\left[\alpha^{\mathrm{m}}\left(\mathbf{r}_{2}\right)\right]^{*} \alpha^{\mathrm{m}}\left(\mathbf{r}_{1}\right)\left[\partial_{x} T_{y y}^{\mathrm{hh}}\left(\mathbf{r}_{1}, \mathbf{r}_{2}\right)\left|H_{y}\right|^{2}+\partial_{x} T_{x x}^{\mathrm{hh}}\left(\mathbf{r}_{1}, \mathbf{r}_{2}\right)\left|H_{x}\right|^{2}\right]^{*} \\
& \left.-\frac{2}{3} k_{0}^{4} \alpha^{\mathrm{e}}\left(\mathbf{r}_{1}\right)\left[\alpha^{\mathrm{m}}\left(\mathbf{r}_{1}\right)\right]^{*}\left[\left[\alpha^{\mathrm{e}}\left(\mathbf{r}_{2}\right) T_{z y}^{\mathrm{he}}\left(\mathbf{r}_{1}, \mathbf{r}_{2}\right)\right]^{*}\left|E_{y}\right|^{2}-\alpha^{\mathrm{m}}\left(\mathbf{r}_{2}\right) T_{z y}^{\mathrm{eh}}\left(\mathbf{r}_{1}, \mathbf{r}_{2}\right)\left|H_{y}\right|^{2}\right]\right\},
\end{aligned}
$$

and the $y$ component can be written as

$$
\begin{aligned}
F_{y}\left(\mathbf{r}_{1}\right)= & \frac{1}{2} \operatorname{Re}\left\{\left[\partial_{y} T_{x y}^{\mathrm{ee}}\right]^{*}\left[\alpha^{\mathrm{e}}\left(\mathbf{r}_{2}\right)\right]^{*} \alpha^{\mathrm{e}}\left(\mathbf{r}_{1}\right) \operatorname{Re}\left[E_{x} E_{y}^{*}\right]+\left[\partial_{y} T_{x y}^{\mathrm{hh}}\right]^{*}\left[\alpha^{\mathrm{m}}\left(\mathbf{r}_{2}\right)\right]^{*} \alpha^{\mathrm{m}}\left(\mathbf{r}_{1}\right) \operatorname{Re}\left[H_{x} H_{y}^{*}\right]\right. \\
& +\left[\partial_{y} T_{x z}^{\mathrm{he}}\right]^{*}\left[\left[\alpha^{\mathrm{m}}\left(\mathbf{r}_{2}\right)\right]^{*} \alpha^{\mathrm{e}}\left(\mathbf{r}_{1}\right)\left\{E_{x}\left[T_{y z}^{\mathrm{eh}} \alpha^{\mathrm{e}}\left(\mathbf{r}_{1}\right) E_{y}\right]^{*}-\left[T_{y z}^{\mathrm{e}} \alpha\left(\mathbf{r}_{2}\right)^{\mathrm{m}} H_{y}\right] H_{x}^{*}\right\}\right. \\
& \left.+\left[\alpha^{\mathrm{e}}\left(\mathbf{r}_{2}\right)\right]^{*} \alpha^{\mathrm{m}}\left(\mathbf{r}_{1}\right)\left\{H_{x}\left[T_{y z}^{\mathrm{eh}} \alpha^{\mathrm{m}}\left(\mathbf{r}_{1}\right) H_{y}\right]^{*}-T_{y z}^{\mathrm{eh}} \alpha^{\mathrm{e}}\left(\mathbf{r}_{2}\right) E_{y} E_{x}^{*}\right\}\right] \\
& \left.+\frac{2}{3} k_{0}^{4} \alpha^{\mathrm{e}}\left(\mathbf{r}_{1}\right)\left[\alpha^{\mathrm{m}}\left(\mathbf{r}_{1}\right)\right]^{*}\left\{E_{x}\left[T_{y z}^{\mathrm{eh}} \alpha^{\mathrm{e}}\left(\mathbf{r}_{2}\right) E_{y}\right]^{*}+T_{y z}^{\mathrm{eh}} \alpha^{\mathrm{m}}\left(\mathbf{r}_{2}\right) H_{y} H_{x}^{*}\right\}\right\} .
\end{aligned}
$$

\section{APPENDIX B: DERIVATIVE OF THE FREE-SPACE SUSCEPTIBILITY TENSOR}

In this section, we give the analytical expressions of the field-susceptibility tensor of homogeneous space and its derivatives, as they are used in this paper. With the notation $\mathbf{a}=\left(x_{2}-x_{1}\right), a=\left|x_{2}-x_{1}\right|, \mathbf{u}=\mathbf{a} / a$, and $y_{1}=y_{2}=z_{1}=$ $z_{2}=0$ we can write

$$
\begin{gathered}
T_{z y}^{\mathrm{eh}}\left(\mathbf{r}_{1}, \mathbf{r}_{2}\right)=\mathbf{u}\left(-\frac{k_{0}^{2}}{a}+i \frac{k_{0}}{a^{2}}\right) e^{i k_{0} a}=-T_{y z}^{\mathrm{eh}}\left(\mathbf{r}_{1}, \mathbf{r}_{2}\right), \\
\partial_{x} T_{x x}^{\mathrm{ee}}\left(\mathbf{r}_{1}, \mathbf{r}_{2}\right)=\mathbf{u}\left[\frac{2 k_{0}^{2}}{a^{2}}+i \frac{6 k_{0}}{a^{3}}-\frac{6}{a^{4}}\right] e^{i k_{0} a}, \\
\partial_{x} T_{y y}^{\mathrm{ee}}\left(\mathbf{r}_{1}, \mathbf{r}_{2}\right)=\mathbf{u}\left[i \frac{k_{0}^{3}}{a}-\frac{2 k_{0}^{2}}{a^{2}}-i \frac{3 k_{0}}{a^{3}}+\frac{3}{a^{4}}\right] e^{i k_{0} a}, \\
\partial_{x} T_{y z}^{\mathrm{eh}}\left(\mathbf{r}_{1}, \mathbf{r}_{2}\right)=\left[i \frac{k_{0}^{3}}{a}-\frac{2 k_{0}^{2}}{a^{2}}-i \frac{4 k_{0}}{a^{3}}\right] e^{i k_{0} a}, \\
\partial_{y} T_{x z}^{\mathrm{eh}}\left(\mathbf{r}_{1}, \mathbf{r}_{2}\right)=\left[-\frac{k_{0}^{2}}{a^{2}}-i \frac{k_{0}}{a^{3}}\right] e^{i k_{0} a}, \\
\partial_{y} T_{x y}^{\mathrm{ee}}=\mathbf{u}\left[\frac{3}{a^{4}}-i \frac{3 k_{0}}{a^{3}}-\frac{k_{0}^{2}}{a^{2}}\right] e^{i k_{0} a}, \\
\partial_{y} T_{x z}^{\mathrm{he}}=\left[\frac{k_{0}^{2}}{a^{2}}+i \frac{k_{0}}{a^{3}}\right] e^{i k_{0} a} .
\end{gathered}
$$

\section{APPENDIX C: COMPUTATION OF THE FIELD AND ITS DERIVATIVE FOR TWO SMALL SPHERES IN INTERACTION: QUADRUPOLAR APPROXIMATION}

It is formally possible to extend the DDA with a multipolar expansion, but in practice this is never done as the computation becomes very tedious, and the same benefits can be achieved by using a finer level of discretization. In this Appendix, we write the DDA up to the quadrupolar order for two small spheres in interaction. Then, the field at position $\mathbf{r}_{1}$ can be written as ${ }^{56,59,60}$

$$
\begin{aligned}
\mathbf{E}\left(\mathbf{r}_{1}\right)= & \mathbf{E}^{\mathrm{inc}}\left(\mathbf{r}_{1}\right)+\mathbf{T}^{\mathrm{ee}}\left(\mathbf{r}_{1}, \mathbf{r}_{2}\right) \alpha^{\mathrm{e}}\left(\mathbf{r}_{2}\right) \mathbf{E}\left(\mathbf{r}_{2}\right) \\
& +\mathbf{T}^{\mathrm{eh}}\left(\mathbf{r}_{1}, \mathbf{r}_{2}\right) \alpha^{\mathrm{m}}\left(\mathbf{r}_{2}\right) \mathbf{H}\left(\mathbf{r}_{2}\right) \\
& +\frac{1}{9}\left[\partial_{i}^{\prime} \mathbf{T}^{\mathrm{ee}}\left(\mathbf{r}_{1}, \mathbf{r}_{2}\right) \alpha_{\mathrm{q}}^{\mathrm{e}}\left(\mathbf{r}_{2}\right) \partial_{i}^{\prime} \mathbf{E}\left(\mathbf{r}_{2}\right)\right. \\
& \left.+\partial_{i}^{\prime} \mathbf{T}^{\mathrm{eh}}\left(\mathbf{r}_{1}, \mathbf{r}_{2}\right) \alpha_{\mathrm{q}}^{\mathrm{m}}\left(\mathbf{r}_{2}\right) \partial_{i}^{\prime} \mathbf{H}\left(\mathbf{r}_{2}\right)\right], \\
\mathbf{H}\left(\mathbf{r}_{1}\right)= & \mathbf{H}^{\mathrm{inc}}\left(\mathbf{r}_{1}\right)+\mathbf{T}^{\mathrm{me}}\left(\mathbf{r}_{1}, \mathbf{r}_{2}\right) \alpha^{\mathrm{e}}\left(\mathbf{r}_{2}\right) \mathbf{E}\left(\mathbf{r}_{2}\right) \\
& +\mathbf{T}^{\mathrm{hh}}\left(\mathbf{r}_{1}, \mathbf{r}_{2}\right) \alpha^{\mathrm{m}}\left(\mathbf{r}_{2}\right) \mathbf{H}\left(\mathbf{r}_{2}\right) \\
& +\frac{1}{9}\left[\partial_{i}^{\prime} \mathbf{T}^{\mathrm{me}}\left(\mathbf{r}_{1}, \mathbf{r}_{2}\right) \alpha_{\mathrm{q}}^{\mathrm{e}}\left(\mathbf{r}_{2}\right) \partial_{i}^{\prime} \mathbf{E}\left(\mathbf{r}_{2}\right)\right. \\
& \left.+\partial_{i}^{\prime} \mathbf{T}^{\mathrm{hh}}\left(\mathbf{r}_{1}, \mathbf{r}_{2}\right) \alpha_{\mathrm{q}}^{\mathrm{m}}\left(\mathbf{r}_{2}\right) \partial_{i}^{\prime} \mathbf{H}\left(\mathbf{r}_{2}\right)\right],
\end{aligned}
$$

where $\partial_{i}^{\prime}$ is the derivative with respect to $\mathbf{r}_{2}$ with a summation over the repeated index $i$ and $\alpha_{\mathrm{q}}$ is the quadrupolar polarizability. ${ }^{57}$ Then, the derivative of the field can be written as

$$
\begin{aligned}
\partial_{j} \mathbf{E}\left(\mathbf{r}_{1}\right)= & \partial_{j} \mathbf{E}^{\mathrm{inc}}\left(\mathbf{r}_{1}\right)+\partial_{j} \mathbf{T}^{\mathrm{ee}}\left(\mathbf{r}_{1}, \mathbf{r}_{2}\right) \alpha^{\mathrm{e}}\left(\mathbf{r}_{2}\right) \mathbf{E}\left(\mathbf{r}_{2}\right) \\
& +\partial_{j} \mathbf{T}^{\mathrm{em}}\left(\mathbf{r}_{1}, \mathbf{r}_{2}\right) \alpha^{\mathrm{m}}\left(\mathbf{r}_{2}\right) \mathbf{H}\left(\mathbf{r}_{2}\right) \\
& +\frac{1}{9}\left[\partial_{j} \partial_{i}^{\prime} \mathbf{T}^{\mathrm{ee}}\left(\mathbf{r}_{1}, \mathbf{r}_{2}\right) \alpha_{\mathrm{q}}^{\mathrm{e}}\left(\mathbf{r}_{2}\right) \partial_{i}^{\prime} \mathbf{E}\left(\mathbf{r}_{2}\right)\right. \\
& \left.+\partial_{j} \partial_{i}^{\prime} \mathbf{T}^{\mathrm{eh}}\left(\mathbf{r}_{1}, \mathbf{r}_{2}\right) \alpha_{\mathrm{q}}^{\mathrm{m}}\left(\mathbf{r}_{2}\right) \partial_{i}^{\prime} \mathbf{H}\left(\mathbf{r}_{2}\right)\right], \\
\partial_{j} \mathbf{H}\left(\mathbf{r}_{1}\right)= & \partial_{j} \mathbf{H}^{\mathrm{inc}}\left(\mathbf{r}_{1}\right)+\partial_{j} \mathbf{T}^{\mathrm{he}}\left(\mathbf{r}_{1}, \mathbf{r}_{2}\right) \alpha^{\mathrm{e}}\left(\mathbf{r}_{2}\right) \mathbf{E}\left(\mathbf{r}_{2}\right) \\
& +\partial_{j} \mathbf{T}^{\mathrm{mm}}\left(\mathbf{r}_{1}, \mathbf{r}_{2}\right) \alpha^{\mathrm{m}}\left(\mathbf{r}_{2}\right) \mathbf{H}\left(\mathbf{r}_{2}\right) \\
& +\frac{1}{9}\left[\partial_{j} \partial_{i}^{\prime} \mathbf{T}^{\mathrm{me}}\left(\mathbf{r}_{1}, \mathbf{r}_{2}\right) \alpha_{\mathrm{q}}^{\mathrm{e}}\left(\mathbf{r}_{2}\right) \partial_{j} \partial_{i}^{\prime} \mathbf{E}\left(\mathbf{r}_{2}\right)\right. \\
& \left.+\partial_{j} \partial_{i}^{\prime} \mathbf{T}^{\mathrm{hh}}\left(\mathbf{r}_{1}, \mathbf{r}_{2}\right) \alpha_{\mathrm{q}}^{\mathrm{m}}\left(\mathbf{r}_{2}\right) \partial_{i}^{\prime} \mathbf{H}\left(\mathbf{r}_{2}\right)\right] .
\end{aligned}
$$

One can notice immediately that the electromagnetic field and its derivative are all computed in a self-consistent way. Obviously, the size of the linear system to solve has now increased to $48 \times 48$. As in our configuration the spheres are under a plane-wave illumination, the linear system can be solved analytically.
${ }^{1}$ A. Ashkin, Phys. Rev. Lett. 24, 156 (1970).

${ }^{2}$ S. Chu, Rev. Mod. Phys. 70, 685 (1998).

${ }^{3}$ C. N. Cohen-Tannoudji, Rev. Mod. Phys. 70, 707 (1998).

${ }^{4}$ W. D. Phillips, Rev. Mod. Phys. 70, 721 (1998).
${ }^{5}$ A. Ashkin, J. M. Dziedzic, J. E. Bjorkholm, and S. Chu, Opt. Lett. 11, 288 (1986).

${ }^{6}$ A. Ashkin, J. M. Dziedzic, and T. Yamane, Nature (London) 330, 769 (1987). 
${ }^{7}$ D. G. Grier, Nature (London) 424, 810 (2003).

${ }^{8}$ K. Dholakiaa, P. Reecea, and M. Gub, Chem. Soc. Rev. 37, 42 (2008).

${ }^{9}$ L. Novotny, R. X. Bian, and X. S. Xie, Phys. Rev. Lett. 79, 645 (1997).

${ }^{10}$ P. C. Chaumet, A. Rahmani, and M. Nieto-Vesperinas, Phys. Rev. B 71, 045425 (2005).

${ }^{11}$ M. Dienerowitz, M. Mazilu, and K. Dholakia, J. Nanophotonics 2, 021875 (2008).

${ }^{12}$ P. C. Chaumet, A. Rahmani, and M. Nieto-Vesperinas, Phys. Rev. Lett. 88, 123601 (2002).

${ }^{13}$ P. C. Chaumet, A. Rahmani, and M. Nieto-Vesperinas, Appl. Opt. 45, 5185 (2006).

${ }^{14}$ A. Rahmani and P. C. Chaumet, Opt. Express 14, 6353 (2006).

${ }^{15}$ M. Barth and O. Benson, Appl. Phys. Lett. 89, 253114 (2006).

${ }^{16}$ M. Righini, A. S.Zelenina, C. Girard, and R. Quidant, Nat. Phys. 3, 477 (2007).

${ }^{17}$ C. A. Mejia, A. Dutt, and M. L. Povinelli, Opt. Express 19, 11422 (2011).

${ }^{18}$ C. A. Mejia, N. Huang, and M. L. Povinelli, Opt. Lett. 37, 3690 (2012).

${ }^{19}$ M. M. Burns, J.-M. Fournier, and J. A. Golovchenko, Phys. Rev. Lett. 63, 1233 (1989)

${ }^{20}$ T. M. Grzegorczyk, B. A. Kemp, and J. A. Kong, Phys. Rev. Lett. 96, 113903 (2006).

${ }^{21}$ S. A. Tatarkova, A. E. Carruthers, and K. Dholakia, Phys. Rev. Lett. 89, 283901 (2002).

${ }^{22}$ M. I. Antonoyiannakis and J. B. Pendry, Phys. Rev. B 60, 2363 (1999).

${ }^{23}$ S. Mohanty, J. Andrews, and P. Gupta, Opt. Express 12, 2746 (2004).

${ }^{24}$ O. Brzobohatý, T. Čižmár, V. Karásek, M. Šiler, K. Dholakia, and P. Zemánek, Opt. Express 18, 25389 (2010).

${ }^{25}$ V. Karèsek, K. Dholakia, and P. Zemànek, Appl. Phys. B 84, 149 (2006).

${ }^{26}$ P. C. Chaumet and M. Nieto-Vesperinas, Phys. Rev. B 64, 035422 (2001).

${ }^{27}$ T. Čižmàr, L. C. D. Romero, K. Dholakia, and D. L. Andrews, J. Phys. B: At., Mol. Opt. Phys. 43, 102001 (2010).

${ }^{28}$ D. Haefner, S. Sukhov, and A. Dogariu, Phys. Rev. Lett. 103, 173602 (2009).

${ }^{29}$ L. Jia and E. L. Thomas, Phys. Rev. B 84, 125128 (2011).

${ }^{30}$ A. Grbic and G. V. Eleftheriades, J. Appl. Phys. 92, 5930 (2002).
${ }^{31}$ N. Engheta and R. W. Ziolkowski, Electromagnetic Metamaterials: Physics and Engineering Explorations (Wiley, New York, 2006).

${ }^{32}$ N. Engheta and R. W. Ziolkowski, IEEE Trans. Microwave Theory Tech. 53, 1535 (2005).

${ }^{33}$ M. Nieto-Vesperinas, R. Gomez-Medina, and J. J. Saenz, J. Opt. Soc. Am. A 28, 54 (2011).

${ }^{34}$ M. Nieto-Vesperinas and J. J. Saenz, Opt. Lett. 35, 4078 (2010).

${ }^{35}$ M. Nieto-Vesperinas, J. J. Sáenz, R. Gómez-Medina, and L. Chantada, Opt. Express 18, 11428 (2010).

${ }^{36}$ P. C. Chaumet, K. Belkebir, and A. Rahmani, Opt. Express 19, 2466 (2011).

${ }^{37}$ A. Dogariu, S. Sukhov, and J. J. Sanez, Nat. Photonics 7, 24 (2013).

${ }^{38}$ P. C. Chaumet, A. Rahmani, A. Sentenac, and G. W. Bryant, Phys. Rev. E 72, 046708 (2005).

${ }^{39}$ J. D. Jackson, Classical Electrodynamics, 2nd ed. (Wiley, New York, 1975).

${ }^{40}$ P. C. Chaumet and C. Billaudeau, J. Appl. Phys. 101, 023106 (2007).

${ }^{41}$ P. C. Waterman, Phys. Rev. D 3, 825 (1971).

${ }^{42}$ P. L. Marston and J. H. Crichton, Phys. Rev. A 30, 2508 (1984).

${ }^{43}$ T. A. Nieminen, Opt. Commun. 235, 227 (2004).

${ }^{44}$ B. T. Draine, Astrophys. J. 333, 848 (1988).

${ }^{45}$ M. A. Yurkin and A. G. Hoekstra, J. Quant. Spectrosc. Radiat. Transfer 106, 558 (2007).

${ }^{46}$ B. T. Draine and J. C. Weingartner, Astrophys. J. 470, 551 (1996).

${ }^{47}$ P. C. Chaumet and M. Nieto-Vesperinas, Phys. Rev. B 61, 14119 (2000).

${ }^{48}$ P. C. Chaumet and M. Nieto-Vesperinas, Phys. Rev. B 62, 11185 (2000).

${ }^{49}$ P. C. Chaumet and A. Rahmani, J. Quant. Spectrosc. Radiat. Transfer 110, 22 (2009).

${ }^{50}$ P. C. Chaumet and A. Rahmani, Opt. Express 17, 2224 (2009).

${ }^{51}$ P. C. Chaumet and A. Rahmani, Opt. Lett. 34, 917 (2009).

${ }^{52}$ K. Jay, P. C. Chaumet, T. N. Langtry, and A. Rahmani, J. Nanophotonics 4, 041583 (2010).

${ }^{53}$ P. C. Chaumet, K. Belkebir, and A. Rahmani, Opt. Express 19, 2466 (2011).

${ }^{54}$ E. E. Radescu and G. Vaman, Phys. Rev. E 65, 046609 (2002).

${ }^{55}$ E. E. Radescu and G. Vaman, Phys. Rev. E 65, 035601 (2002).

${ }^{56}$ P. C. Chaumet, A. Rahmani, F. de Fornel, and J.-P. Dufour, Phys. Rev. B 58, 2310 (1998).

${ }^{57}$ R. Rojas and F. Claro, Phys. Rev. B 34, 3730 (1986).

${ }^{58}$ K. Bonin, B. Kourmanov, and T. Walker, Opt. Express 10, 984 (2002).

${ }^{59}$ C. Girard and A. Dereux, Phys. Rev. B 49, 11344 (1994).

${ }^{60}$ A. D. Buckingham, Adv. Chem. Phys. 12, 107 (1967). 